Pat 1

\begin{tabular}{ccccccccc}
\hline \multicolumn{10}{c}{ Time } \\
\hline Tropism & 0 & 12 & 23 & 35 & 44 & 50 & 65 & 77 \\
\hline R5 & 0.25 & - & - & - & - & - & - & - \\
\hline X4 & 0.75 & - & - & - & - & - & - & - \\
\hline HIV VL & 4.41 & $<1.7$ & $<1.7$ & $<1.7$ & $<1.7$ & $<1.7$ & $<1.7$ & $<1.7$ \\
\hline CD4+ & 307 & 17 & 86 & 161 & 202 & 332 & 538 & 400 \\
\hline
\end{tabular}

Pat 5

\begin{tabular}{cccccccc}
\hline \multicolumn{7}{c}{ Time } \\
\hline Tropism & 0 & 12 & 24 & 48 & 57 & 71 & 83 \\
\hline R5 & 1.00 & - & - & - & - & - & - \\
\hline X4 & 0.00 & - & - & - & - & - & - \\
\hline HIV VL & 3.80 & $<1.7$ & $<1.7$ & $<1.7$ & $<1.7$ & $<1.7$ & $<1.7$ \\
\hline CD4+ & NA & NA & NA & NA & NA & NA & NA \\
\hline
\end{tabular}

\section{Pat 8}

\begin{tabular}{ccccccc}
\hline \multicolumn{7}{c}{ Time } \\
\hline Tropism & 0 & 24 & 34 & 47 & 71 & 83 \\
\hline R5 & 0.98 & - & - & - & - & - \\
\hline X4 & 0.02 & - & - & - & - & - \\
\hline HIV VL & 4.52 & $<1.7$ & $<1.7$ & $<1.7$ & $<1.7$ & $<1.7$ \\
\hline CD4+ & NA & NA & NA & NA & NA & NA \\
\hline
\end{tabular}

\section{Pat 27}

\begin{tabular}{|c|c|c|c|c|}
\hline & \multicolumn{4}{|c|}{ Time } \\
\hline Tropism & 0 & 13 & 49 & 59 \\
\hline R5 & 0.04 & - & - & - \\
\hline $\mathrm{X} 4$ & 0.96 & - & - & - \\
\hline HIV VL & 5.37 & $<1.7$ & $<1.7$ & $<1.7$ \\
\hline CD4+ & 143 & 136 & 130 & 149 \\
\hline
\end{tabular}

\section{Pat 3}

\begin{tabular}{cccccccc}
\hline \multicolumn{7}{c}{ Time } \\
\hline Tropism & 0 & 7 & 12 & 25 & 34 & 60 & 71 \\
\hline R5 & 0.96 & 0.97 & 0.95 & - & - & 0.96 & 0.96 \\
\hline X4 & 0.04 & 0.03 & 0.05 & - & - & 0.04 & 0.04 \\
\hline HIV VL & 5.38 & 5.70 & 3.57 & $<1.7$ & $<1.7$ & 3.04 & 3.56 \\
\hline CD4+ & NA & NA & 45 & 397 & 603 & 69 & 68 \\
\hline
\end{tabular}

\section{Pat 26}

\begin{tabular}{ccccccc}
\hline \multicolumn{7}{c}{ Time } \\
\hline Tropism & 0 & 24 & 48 & 59 & 62 & 71 \\
\hline R5 & 0.98 & - & - & - & - & - \\
\hline X4 & 0.02 & - & - & - & - & - \\
\hline HIV VL & 4.52 & $<1.7$ & $<1.7$ & $<1.7$ & $<1.7$ & $<1.7$ \\
\hline CD4+ & 294 & 357 & 514 & 790 & 722 & 792 \\
\hline
\end{tabular}

Pat 28

\begin{tabular}{cccccccc}
\hline \multicolumn{7}{c}{ Time } \\
\hline Tropism & 0 & 24 & 27 & 41 & 65 \\
\hline R5 & 1.00 & - & - & - & - & - \\
\hline X4 & 0.00 & - & - & - & - \\
\hline HIV VL & 5.37 & NA & $<1.7$ & $<1.7$ & $<1.7$ \\
\hline CD4+ & 305 & 256 & 272 & 458 & 479 \\
\hline
\end{tabular}

\section{Pat 6}

\begin{tabular}{ccccccccc}
\hline \multicolumn{10}{c}{ Time } \\
\hline Tropism & -12 & 0 & 7 & 18 & 20 & 37 & 79 & 91 \\
\hline R5 & - & 0.97 & 0.97 & - & - & - & 0.97 & 0.99 \\
\hline X4 & - & 0.03 & 0.03 & - & - & - & 0.03 & 0.01 \\
\hline HIV VL & $<1.7$ & 4.10 & 4.58 & 2.14 & 1.78 & $<1.7$ & 3.15 & 2.33 \\
\hline CD4+ & NA & NA & NA & NA & NA & NA & NA & NA \\
\hline
\end{tabular}

\section{Pat 7}

\begin{tabular}{cccccccccc}
\hline \multicolumn{10}{c}{ Time } \\
\hline Tropism & 0 & 36 & 46 & 57 & 69 & 83 & 93 & 108 & 114 \\
\hline R5 & 0.82 & 0.97 & - & - & - & 0.96 & 0.81 & - & - \\
\hline X4 & 0.18 & 0.03 & - & - & - & 0.04 & 0.19 & - & - \\
\hline HIV VL & 3.59 & 3.96 & $<1.7$ & $<1.7$ & $<1.7$ & 4.72 & 3.73 & 2.05 & $<1.7$ \\
\hline CD4+ & NA & 236 & 263 & 240 & 457 & 388 & 379 & 314 & NA \\
\hline
\end{tabular}

Pat 9

\begin{tabular}{cccccc}
\hline \multicolumn{7}{c}{ Time } \\
\hline Tropism & 0 & 10 & 48 & 84 & 95 \\
\hline R5 & 0.19 & - & 0.87 & 0.82 & 0.72 \\
\hline X4 & 0.81 & - & 0.13 & 0.08 & 0.28 \\
\hline HIV VL & 4.7 & 3.11 & 4.48 & $>5.7$ & 5.67 \\
\hline CD4+ & NA & 525 & NA & 91 & 127 \\
\hline
\end{tabular}

\section{Pat 17}

\begin{tabular}{ccccccccc}
\hline \multicolumn{10}{c}{ Time } \\
\hline Tropism & 0 & 11 & 36 & 48 & 55 & 62 & 73 & 84 \\
\hline R5 & 0.86 & - & - & - & - & - & 0.96 & - \\
\hline X4 & 0.14 & - & - & - & - & - & 0.04 & - \\
\hline HIV VL & 3.94 & 4.5 & 4.8 & $<1.7$ & $<1.7$ & $<1.7$ & $<1.7$ & $<1.7$ \\
\hline CD4+ & 624 & 699 & 688 & NA & 759 & NA & 1384 & 1341 \\
\hline
\end{tabular}

\section{Pat 18}

\begin{tabular}{cccccccc}
\hline \multicolumn{7}{c}{ Time } \\
\hline Tropism & 0 & 24 & 48 & 63 & 73 & 87 & 108 \\
\hline R5 & 0.96 & - & - & - & - & - & - \\
\hline X4 & 0.04 & - & - & - & - & - & - \\
\hline HIV VL & 5.33 & $<1.7$ & NA & NA & $<1.7$ & 3.55 & NA \\
\hline CD4+ & 200 & 306 & 463 & 740 & 619 & 573 & 651 \\
\hline
\end{tabular}


Pat 21

\begin{tabular}{ccccccccc}
\hline \multicolumn{10}{c}{ Time } \\
\hline Tropism & -24 & 0 & 12 & 19 & 29 & 37 & 52 & 60 \\
\hline R5 & - & 1.00 & - & - & 1.00 & 1.00 & - & - \\
\hline X4 & - & 0.00 & - & - & 0.00 & 0.00 & - & - \\
\hline HIV VL & $<1.7$ & 1.98 & 1.90 & $<1.7$ & 5.15 & 4.14 & $<1.7$ & $<1.7$ \\
\hline CD4+ & NA & NA & NA & NA & NA & NA & NA & NA \\
\hline
\end{tabular}

Pat 23

\begin{tabular}{cccccc}
\hline \multicolumn{7}{c}{ Time } \\
\hline Tropism & -36 & 0 & 8 & 23 & 39 \\
\hline R5 & - & 0.28 & - & - & - \\
\hline X4 & - & 0.72 & - & - & - \\
\hline HIV VL & $<1.7$ & 5.05 & 1.90 & $<1.7$ & $<1.7$ \\
\hline CD4+ & NA & NA & NA & NA & NA \\
\hline
\end{tabular}

\section{Pat 24}

\begin{tabular}{ccccc}
\hline \multicolumn{5}{c}{ Time } \\
\hline Tropism & 0 & 24 & 48 & 54 \\
\hline R5 & 1.00 & 1.00 & 1.00 & 1.00 \\
\hline X4 & 0.00 & 0.00 & 0.00 & 0.00 \\
\hline HIV VL & 3.96 & $>5.70$ & 4.00 & 3.96 \\
\hline CD4+ & NA & 107 & NA & 81 \\
\hline
\end{tabular}

Pat 13

\begin{tabular}{cccccc}
\hline \multicolumn{7}{c}{ Time } \\
\hline Tropism & 0 & 30 & 39 & 53 & 64 \\
\hline R5 & - & 1.00 & - & 1.00 & - \\
\hline X4 & - & 0.00 & - & 0.00 & - \\
\hline HIV VL & 3.61 & 2.4 & 1.90 & 4.34 & 1.85 \\
\hline CD4+ & 292 & NA & 139 & NA & 154 \\
\hline
\end{tabular}

\section{Pat 25}

\begin{tabular}{cccccccccc}
\hline & \multicolumn{10}{c}{ Time } \\
\hline Tropism & 0 & 8 & 31 & 40 & 53 & 71 & 83 & 88 & 100 \\
\hline R5 & 0.62 & 0.66 & 0.00 & 0.58 & - & - & - & - & - \\
\hline X4 & 0.38 & 0.34 & 1.00 & 0.42 & - & - & - & - & - \\
\hline HIV VL & 5.51 & 5.70 & $<1.7$ & $<1.7$ & 2.39 & $<1.7$ & $<1.7$ & $<1.7$ & $<1.7$ \\
\hline CD4+ & 393 & 448 & 524 & 891 & NA & 485 & 580 & 743 & 858 \\
\hline
\end{tabular}

\section{Pat 15}

\begin{tabular}{ccccccccc}
\hline \multicolumn{8}{c}{ Time } \\
\hline Tropism & 0 & 12 & 27 & 32 & 45 & 54 & 65 & 79 \\
\hline R5 & - & - & 0.96 & 0.97 & 0.97 & 0.97 & - & - \\
\hline X4 & - & - & 0.04 & 0.03 & 0.03 & 0.03 & - & - \\
\hline HIV VL & 6.99 & 6.57 & 7.10 & 6.78 & $>7.6$ & $>7.6$ & 7.23 & 6 \\
\hline CD4+ & 790 & 1000 & 532 & 864 & 623 & 628 & NA & 803 \\
\hline
\end{tabular}

Dash: no data. NA: not available.

\section{Pat 14}

\begin{tabular}{cccccccc}
\hline \multicolumn{7}{c}{ Time } \\
\hline Tropism & 0 & 9 & 23 & 37 & 45 & 51 & 63 \\
\hline R5 & - & 1.00 & - & 0.09 & 0.14 & - & - \\
\hline X4 & - & 0.00 & - & 0.91 & 0.86 & - & - \\
\hline HIV VL & $<1.70$ & $<1.70$ & $<1.70$ & 5.46 & $<1.70$ & $<1.70$ & $<1.70$ \\
\hline CD4+ & NA & NA & NA & NA & NA & NA & NA \\
\hline
\end{tabular}

